\title{
15. \\ Ueber das Princip der kleinsten Wirkung.
}

(Principe de la moindre action.)

(Von Herm J. Zech, theol. cand. zu Tübingen.)

I. Das Princip der kleinsten Wirkung hat zuerst Euler in seiner Methodus inveniendi lineas curvas, maximi minimive proprielate gaudentes, Laus. et Genev. 1744 im zweiten Anhange "de motu projectorum in medio non resistente per methodum maximorum ac minimorum determinando" aufgestellt, ohne jedoch einen eigentlichen Beweis davon zu geben; was auch bei dem damaligen Zustande der Analysis nicht wohl möglich war. Euler begnügte sich damit, a. a. 0 . und in den Méin. de lacad. roy. de Berlin 1748, pag.149 sqq., sein Princip zur Auflösung verschiedener Aufgaben aus der Mechanik zu benutzen und aus der Uebereinstimmung der gefundenen Resultate mit dem auf anderem Wege Constatirten auf die Richtigkeit desselben zu schliefsen. Lagrange, in der 1788 erschienenen ersten Ausgabe seiner Mécaniqne analytique, gab dem Euler'schen Princip den Namen des Princips der kleinsten Wirkung, nach einem dem Euler'schen ähnlichen, aber weniger bedeutenden und allgemeinen Princip der Mechanik, welches Maupertuis unter diesem Namen im Jahr 1744 der Pariser Akademie vorgelegt hatte, dehnte dasselbe, während es Euler nur in Beziehung auf die Bewegung eines einzelnen Körpers aufgestellt hatte, auf ein beliebiges System von Körpern aus und gab zuerst einen analytischen Beweis davon, indem er es mit Hülfe der Variationsrechnung aus den bekannten Gleichungen der Bewegung ableitete. In dieser Erweiterung ging das Princip wohl in alle neuere Lehrbücher der Mechanik über, aber melır nur als metaphysisch merkwürdiges Resultat mathematischer Formelı, als um weitere Folgerungen daraus abzuleiten.

Der gewöhnliche Ausdruck des Princips der kleinsten Wirkung ist folgender. Wenn auf einen Körper blof Centralkräfte wirken, überhaupt wenn $\boldsymbol{X} \boldsymbol{d} \boldsymbol{x}+\boldsymbol{Y} \boldsymbol{d} \boldsymbol{y}+\boldsymbol{Z} \boldsymbol{d} \boldsymbol{z}$, wo $\boldsymbol{X}, \boldsymbol{Y}, \boldsymbol{Z}$ die Composanten der auf den Körper wirkenden Kräfte parallel mit den drei Coordiualen-Axen be- 
zeichnen, ein vollständiges Differential ist, so ist die von dem Körper frei beschriebene Bahn und seine Bewegung in derselben so beschaffen, dafs, wenn $s$ den durchlaufenen Weg, $v$ die Geschwindigkeit und $t$ die Zeit bezeichnet, $\int d s . v=\int d t . v^{2}$ ein Maximum oder Minimum wird, in Beziehung auf alle Curven, welche der Körper zwischen denselben Endpuncten hätte beschreiben können. Wäre der Körper genöthigt, sich auf einer gegebenen Fläche zu bewegen, so ist $\int d s . v$ oder $\int d t . v^{2}$ immer noch ein Maximum oder Minimum, aber nur in Beziehung auf alle Curven, welche der Körper zwischen denselben Endpuncten auf der gegebenen Fläche hätte beschreiben können. Bei der freien Bewegung eines Systems von Körpern ist, wenn dieselben nur durch ihre gegenseitigen Anziehungskräfte und durch Centralkräfte getrieben werden, überhaupt wenn $\sum m(X d x+Y d y+Z d z)$ ein vollständiges Differential ist, die Summe der Producte aus der Masse jedes einzelnen Körpers in das ihm entsprechende $\int d s . v$ oder $\int d t . v^{2}$, d. h. die Summe der lebendigen Kräfte, ein Maximum oder ein Minimum : wiederum vorausgesetzt, dafs die Anfạngsund die Endpuncte sämmtlicher Bahnen, welche die einzelnen Körper beschreiben, als gegeben angesehen werden. Euler, Laplace und Poisson sagen ausdrücklich: ein Maximum oder Minimum unter allen Bahnen, welche zwischen denselben Eudpuncten enthalten sind; und wenn Andere, wie Lagrange, Pontécoulant etc. nur überhaupt von einem Maximum oder Minimum sprechen, so rührt dies wohl nur davon her, dafs sie jede weitere Bemerkung für überflüssig hielten, und nicht davon, dafs sie anderer Meinung waren; was sie sonst gewifs ausdrücklich gesagt hätten. Der Beweis des Princips ist bei Verschiedenen verschieden; alle Modificationen desselben aber laufen darauf hinaus, dafs mit Hülfe der bekannten Formeln der Dynamik, nämlich

$$
\begin{aligned}
& \text { 1. }\left(\frac{d s}{d t}\right)^{2}=v^{2}=\left(\frac{d x}{d t}\right)^{2}+\left(\frac{d y}{d t}\right)^{2}+\left(\frac{d z}{d t}\right)^{2}, \\
& \text { 2. } v^{2}=C+2 \int(X d x+Y d y+Z d z), \\
& \text { 3. } \quad X=\frac{d^{2} x}{d t^{2}} ; \quad Y=\frac{d^{2} y}{d t^{2}} ; \quad Z=\frac{d^{2} z}{d t^{2}}
\end{aligned}
$$

gezeigt wird, dafs 


$$
\delta \int d s . v=\delta \int d t . v^{2}=\frac{d x}{d t} \delta x+\frac{d y}{d t} \delta y+\frac{d z}{d t} \delta z+\text { const. }
$$

ist. Für die beiden Grenzen der Bewegung wird dann weiter geschlossen, es sei, da nach der Voraussetzung $\delta x=0, \delta y=0, \delta z=0$ ist:

$$
\delta \int d s . v=\delta \int d t \cdot v^{2}=0
$$

und folglich $\int d s . v=\int d t . v^{2}$ ein Maximum oder Minimum: bei der ganz freien Bewegung natürlich das letztere, weil hier die Nachbarcurven unbeschränkt sind.

II. Dafs nun aber dieses Princip der kleinsten Wirkung, wenigstens in der Allgemeinheit, in welcher es gewöhnlich ausgesprochen wird, nicht stattfinde, kann durch folgendes Beispiel gezeigt werden:

Wenn ein Körper mit einer beliebigen Geschwindigkeit in einer gegen den Horizont schiefen Richtung in die Höhe geworfen wird, so beschreibt er bei der freien Bewegung in luftleeren Raume eine Parabel. Wäre er nun aber gezwungen, mit derselben Anfangsgeschwindigkeit in derselben Richtung geworfen, eine Cycloüde, welche jene Parabel im Anfangspuncte der Bewegung und noch in einem andern Puncte berührt, zu durchlaufen, so würde aus dem Princip der kleinsten Wirkung folgen, dafs das zwischen den beiden Berührungspuncten genommene $\int d s . v$ oder $\int d t . v^{2}$ im letztern Fall gröfser sei, als im erstern. Ob sich dies wirklich so verhalte, ist zu untersuchen.

Die Coordinaten-Axen seien in der Vertical-Ebene, in welcher die Anfangsgeschwindigkeit und daher auch beide Bahnen liegen, so gelegt, dafs der Ursprung derselben mit dem Anfangspuncte der Bewegung zusammenfällt und die Axe der $x$ horizontal und die der $y$ vertical ist, und zwar letztere mit ihrer positiven Seite der Richtung der Schwere entgegenläuft. Die Anfangsgeschwindigkeit, mit welcher der Körper geworfen wird, sei $=a$; der Winkel, welchen die Richtung derselben, mithin auch die gemeinschaftliche Tangente beider Curven im Anfangspuncte, mit der Axe der $x$ macht, $=\alpha$; die Schwere endlich $=g$ : so hat man zur Bestimmung der Bewegung in der Cycloïde, aulser den Gleichungen der Cycloïle selbst, die für jede freie und unfreie, durch die Schwere hervorgebrachte Bewegung geltende Gleichung:

$$
v^{2}=a^{2}-2 g y
$$


woraus

$$
\text { 1. } \int d s \cdot v=\int d s \cdot V\left(a^{2}-2 g y\right)=\int d y \cdot \frac{d s}{d y} \cdot V\left(a^{2}-2 g y\right)
$$

folgt. Ist nun die Basis der Cycloïde horizontal, also parallel mit der Axe $\operatorname{der} x, \boldsymbol{r}$ der Halbmesser des Erzeugungskreises und $\vartheta$ der Wälzungswinkel, und werden durch, den Anfangspunct der Cycloïde neue, mit den alten parallele Coordinaten - Axen gelegt, so sind, wenn die neuen Coordinaten durch $x^{\prime}, y^{\prime}$ bezeichnet werden, die Gleichungen der Cycloïde, auf diese neuen Axen bezogen, folgende:

$$
\text { 2. } x^{\prime}=r(\vartheta-\sin \vartheta) \quad \text { und } \quad y^{\prime}=r(1-\cos \vartheta) \text {. }
$$

Um die Gleichungen der Cyclö̈de für das ursprüngliche Coordinatensystem zu finden, müssen die auf das neue System bezogenen Coordinaten $\left(x^{\prime}\right)$ und $\left(y^{\prime}\right)$ des Anfangspunctes, der Bewegung aus der Bedingung bestimmt werden, dafs die Tangente an diesen Punct der Cycloïde mit der Axe der $x$, also auch der der $x^{\prime}$, den Winkel $\alpha$ macht, und dann ist

$$
x^{\prime}=x+\left(x^{\prime}\right) \text { und } y^{\prime}=y+\left(y^{\prime}\right) \text {. }
$$

Nun folgt aus den Gleichungen (2.), wenn man differentiirt,

$$
\frac{d x^{\prime}}{d \vartheta}=r(1-\cos \vartheta) ; \quad \frac{d y^{\prime}}{d \vartheta}=r \cdot \sin \vartheta
$$

folglich

$$
\frac{d y^{\prime}}{d x^{\prime}}=\frac{\sin \vartheta}{1-\cos ף}=\operatorname{cotang} \frac{1}{2} \vartheta
$$

Für den Punct $\left(x^{\prime}\right),\left(y^{\prime}\right)$ ist also

mithin aus (2.)

$$
\text { cotang } \frac{1}{2} \vartheta=\operatorname{tang} \alpha, \quad \vartheta=\pi-2 \alpha,
$$

$$
\left(x^{\prime}\right)=r(\pi-2 \alpha-\sin 2 \alpha) ; \quad\left(y^{\prime}\right)=r(1+\cos 2 \alpha)=2 r \cdot \cos ^{2} \alpha .
$$

Die Gleichungen der Cyclö̈e, auf die ursprünglichen Coordinaten-Axen bezogen, sind also

3. $x+r(\pi-2 \alpha-\sin 2 \alpha)=r(\vartheta-\sin \vartheta)$ und $y+2 r \cdot \cos ^{2} \alpha=r(1-\cos \vartheta)$. Differentiirt man beide Gleichungen nach $\vartheta$, so findet sich

folglich

$$
\begin{aligned}
\frac{d x}{d \vartheta} & =r(1-\cos \vartheta)=y+2 r \cdot \cos ^{2} \alpha \\
\frac{d y}{d \vartheta} & =r \cdot \sin \vartheta=\checkmark\left\{r^{2}-\left[r-\left(y+2 r \cdot \cos ^{2} \alpha\right)\right]^{2}\right\} \\
& =r\left\{\left[r+r-\left(y+2 r \cdot \cos ^{2} \alpha\right)\right]\left(r-r+y+2 r \cdot \cos ^{2} \alpha\right)\right\} \\
& =r\left\{\left(2 r \cdot \sin ^{2} \alpha-y\right)\left(2 r \cdot \cos ^{2} \alpha+y\right)\right\},
\end{aligned}
$$


16̆. Zcch, über das Princip der kleinsten Wirkung.

$$
\begin{aligned}
& \frac{d x}{d y}=V\left(\frac{2 r \cdot \cos ^{2} \alpha+y}{2 r \cdot \sin ^{2} \alpha-y}\right) \\
& \frac{d s}{d y}=V\left(1+\left(\frac{d x}{d y}\right)^{2}\right)=V\left(1+\frac{2 r \cdot \cos ^{2} \alpha+y}{2 r \cdot \sin ^{2} \alpha-y}\right)=V\left(\frac{2 r}{2 r \cdot \sin ^{2} \alpha-y}\right) .
\end{aligned}
$$

Durch diesen Werth von $\frac{d s}{d y}$ geht die (1.) über in

$$
\text { 4. } \begin{aligned}
\int d s \cdot v & =\int d y \cdot V\left(\frac{2 r}{2 r \cdot \sin ^{2} a-y}\right) \cdot r\left(a^{2}-2 g y\right) \\
& =2 r(g r) \cdot \int d y \cdot \sqrt{ }\left(\frac{\frac{a^{2}}{2 g}-y}{2 r \cdot \sin ^{2} a-y}\right) .
\end{aligned}
$$

Um integriren zu können, setze man

so wiird

$$
\text { 5. } \left.\quad \sqrt{\left(\frac{a^{2}}{2 g}-y\right.} \frac{\sin ^{2} \alpha-y}{2 r \cdot \sin ^{2}}\right)=z,
$$

folglich

$$
\begin{aligned}
\frac{a^{2}}{2 g}-y & =\left(2 r \cdot \sin ^{2} \alpha-y\right) z^{2} \\
y & =\frac{2 r \cdot \sin ^{2} \alpha \cdot z^{2}-\frac{a^{2}}{2 g}}{z^{2}-1}=\frac{1}{2 g} \cdot \frac{4 g r \cdot \sin ^{2} \alpha \cdot z^{2}-a^{2}}{z^{2}-1}, \\
\frac{d y}{d z} & =\frac{1}{2 g} \cdot \frac{8 g r \cdot \sin ^{2} \alpha \cdot z\left(z^{2}-1\right)-2 z\left(4 g r \cdot \sin ^{2} \alpha \cdot z^{2}-a^{2}\right)}{\left(z^{2}-1\right)^{2}} \\
& =\frac{z}{g} \cdot \frac{a^{2}-4 g r \cdot \sin ^{2} \alpha}{\left(z^{2}-1\right)^{2}}
\end{aligned}
$$

$$
\begin{aligned}
\int d s . v & =2 V \frac{r}{g} \cdot\left(a^{2}-4 g r \cdot \sin ^{2} \alpha\right) \cdot \int d z \frac{z^{2}}{\left(z^{2}-1\right)^{2}} \\
& =2 V \frac{r}{g}\left(a^{2}-4 g r \cdot \sin ^{2} \alpha\right)\left(-\frac{z}{2\left(z^{2}-1\right)}+\frac{1}{2} \int d z \frac{1}{z^{2}-1}\right),
\end{aligned}
$$

6. $\int d s \cdot v=-\sqrt{ } \frac{r}{g}\left(a^{2}-4 g r \cdot \sin ^{2} \alpha\right)\left(\frac{z}{z^{2}-1}+\frac{1}{2} l \frac{z+1}{z-1}\right)+$ const.

Da die Basis der Cycloïde horizontal vorausgesetzt worden ist und die Axe der Parabel der Natur der Sache nach vertical sein mufs, so folgt, dafs die beiden Berührungspuncte beider Curven in gleicher Höhe über dem Horizonte liegen müssen, mithin gleiche Ortinaten haben. Um also die Constante in (6.) zu bestimmen, müfste der Ausdruck rechts zwischen zwei gleichen Grenzen genommen werden. Da dieses aber zu keinem Resultate führen würde, nimmt man, da auf beiden Seiten der durch den höchsten Punct der Cycloïde gehenden Verticalen die $y$, also auch die $z$ genau dieselben sind, obigen Ausdruck stalt einmal vom Anfangs- bis zum End- 
puncte der Bahn, zweimal vom Anfangspuncte der Bewegung bis zum höchsten Punct der Cycloïde. Für jenen Punct ist

$$
y=0, \quad \text { also aus (5.) } \quad z=\frac{a}{2 \sqrt{(g r) \cdot \sin \alpha}} \text {. }
$$

Für den höchsten Punct der Cyclö̈de ist $\vartheta=\pi$, also aus (3.)

mithin

$$
y=2 r_{0} \sin ^{2} \alpha \text {. und daher } \approx=\infty,
$$

$$
\int d s . v=-2 \sqrt{\frac{r}{g}} \cdot\left(u^{2}-4 g r \cdot \sin ^{2} a\right)\left(\frac{z}{z^{2}-1}+\frac{z}{2} l \frac{z+1}{z-1}\right)_{\infty \div \frac{a}{2 v}(\operatorname{grr}) \cdot \sin a},
$$

oder wegen

$$
\begin{gathered}
\frac{\infty}{\infty^{2}-1}=\frac{1}{\infty-\frac{1}{\infty}}=0, \quad \text { und } \quad l \frac{\infty+1}{\infty-1}=l \frac{1+\frac{1}{\infty}}{1-\frac{1}{\infty}}=l 1=0, \\
\int d s . v=2 \sqrt{\frac{r}{g}}\left(a^{2}-4 g r \cdot \sin ^{2} \alpha\right)\left(\frac{2 a \sqrt{ }(g r) \cdot \sin \alpha}{a^{2}-4 g r \cdot \sin ^{2} \alpha}+\frac{1}{2} l \frac{a+2 \sqrt{ }(g r) \cdot \sin \alpha}{a-2 \sqrt{ }(g r) \cdot \sin \alpha}\right), \\
\text { 7. } \int d s . v=4 a r \cdot \sin \alpha+\sqrt{\frac{r}{g}}\left(a^{2}-4 g r \cdot \sin ^{2} \alpha\right) l \frac{a+2 \sqrt{ }(g r) \cdot \sin \alpha}{a-2 \sqrt{ }(g r) \cdot \sin \alpha}
\end{gathered}
$$

Für die freie Bewegung in der Parabel hat man folgende Gleichungen:

$$
\begin{array}{rlrl}
\text { 8. } \quad \frac{d^{2} x}{d t^{2}} & =0, \quad \frac{d^{2} y}{d t^{2}} & =-g, \\
\text { 9. } \quad \frac{d x}{d t} & =a \cdot \cos a, \quad \frac{d y}{d t} & =a \cdot \sin \alpha-g t, \\
\text { 10. } \quad x & =a \cdot \cos a \cdot t \quad y & =a \cdot \sin a \cdot t-\frac{1}{2} \cdot g t^{2}, \\
v^{2} & =\left(\frac{d x}{d t}\right)^{2}+\left(\frac{d y}{d t}\right)^{2}=a^{2}-2 a g \sin a t+g^{2} t^{2},
\end{array}
$$

11. $\int d t \cdot v^{2}=a^{2} \cdot t-a g \cdot \sin a \cdot t^{2}+\frac{1}{3} g^{2} t^{3}+$ const.

Dieses Integral mufs von $t=0$ bis zu demjenigen $t$ genommen werden, welches dem Endpuncte der Bahn entspricht. Die Ordinate dieses Punctes aber ist, wie wir oben gesehen haben, gleich der Ordinate des Anfangspunctes, also $=0$, und dazu giebt die (10)

milbin

$$
t=\frac{2 a \cdot \sin \alpha}{g}
$$

12. $\int d t \cdot v^{2}=\frac{2 a^{3} \cdot \sin \alpha}{g}-\frac{4 a^{3} \cdot \sin ^{3} \alpha}{g}+\frac{8}{3} \cdot \frac{a^{3} \cdot \sin ^{3} \alpha}{g}=\frac{a^{3}}{y} \sin \alpha\left(2-\frac{4}{3} \sin ^{2} \alpha_{3}\right)$. Damit nun aber wirklich die Parabel und die Cycloïde zum zweiten Male sich berühren, müssen die Abscissen des höchsten Punctes der Cycloïde und des höchsten Punctes der Parabel einander gleich sein; wodurch man eine 
Gleichung zur Bestimmung von $a$ bekommt. Es ist aber für den höchsten Punct der Cycloïde $\vartheta=\pi$, also aus (3.)

$$
x=r(2 \alpha+\sin 2 \alpha) \text {. }
$$

Für den höchsten Punct der Parabel hat man

und dazu aus (10.)

$$
\frac{d y}{d t}=0, \quad \text { also aus (9.) } \quad t=\frac{a \cdot \sin \alpha}{g}
$$

Dies giebt die Gleichung

$$
x=\frac{a^{2} \cdot \sin \alpha \cdot \cos \alpha}{g}=\frac{a^{2} \cdot \sin 2 \alpha}{2 y} .
$$

$$
\begin{aligned}
& r(2 \alpha+\sin 2 \alpha)=\frac{a^{2} \cdot \sin 2 \alpha}{2 g}, \\
& \text { 13. } \quad a^{2}=\frac{4 g r(2 \alpha+\sin 2 \alpha)}{2 \sin 2 \alpha} .
\end{aligned}
$$

Dieser Werth für $a$ in (7.) und (12.) substituirt, giebt für die Cycloïde 14. $\int d s . v=8 r(g r)^{\frac{1}{2}}\left\{\sin \alpha\left(\frac{2 \alpha+\sin 2 \alpha}{2 \sin 2 \alpha}\right)^{\frac{1}{2}}\right.$

und für die Parabel

$$
\left.+\frac{1}{2}\left(\frac{2 \alpha+\sin 2 \alpha}{2 \sin 2 \alpha}-\sin ^{2} \alpha\right) l \frac{\left(\frac{2 \alpha+\sin 2 \alpha}{2 \sin 2 \alpha}\right)^{\frac{\alpha}{2}}+\sin \alpha}{\left(\frac{2 \alpha+\sin 2 \alpha}{2 \sin 2 \alpha}\right)^{\frac{1}{2}}-\sin \alpha}\right\}
$$

$$
\text { 15. } \int d s . v=8 r(g r)^{\frac{1}{2}} \sin \alpha\left(2-\frac{4}{3} \sin ^{2} \alpha\right)\left(\frac{2 \alpha+\sin 2 \alpha}{2 \sin 2 \alpha}\right)^{\frac{3}{2}}
$$

Um also das Princip der kleinsten Wirkung zu prüfen, braucht man nur

$$
\sin \alpha\left(\frac{2 \alpha+\sin 2 \alpha}{2 \sin 2 \alpha}\right)^{\frac{1}{2}}+\frac{1}{2}\left(\frac{2 \alpha+\sin 2 \alpha}{2 \sin 2 \alpha}-\sin ^{2} \alpha\right) l \frac{\left(\frac{2 \alpha+\sin 2 \alpha}{2 \sin 2 \alpha}\right)^{\frac{1}{2}}+\sin \alpha}{\left(\frac{2 \alpha+\sin 2 \alpha}{2 \sin 2 \alpha}\right)^{\frac{1}{2}}-\sin \alpha}=A
$$

und

$$
2 \sin \alpha\left(1-\frac{2}{3} \sin ^{2} \alpha\right)\left(\frac{2 \alpha+\sin 2 \alpha}{2 \sin 2 \alpha}\right)^{\frac{3}{2}}=\boldsymbol{B}
$$

für verschiedene Werthe von $a$ zu berechnen und zu sehen, ob immer $\boldsymbol{A}>\boldsymbol{B}$ ist. Allein man findet für
$\alpha=30^{\prime \prime}$
$45^{\circ}$
$60^{\circ}$
$75^{\circ}$
$\boldsymbol{A}=0,9677214$
1,3758234
1,8968039
3,047466 ,
$\boldsymbol{B}=0,967445$
1,373979
1,935173
4,020396;

woraus die Richtigkeit der obigen Behauptung von selbst erhellt.

III. Sieht man nun nach dem Beweise, ob etwa in diesem ein verborgener Fehler stecke, der das Resultat erklärte, so fragt sich zu- 
erst: welches ist die unabhängige Veränderliche, nach welcher die Variationen von $\int d s . v$ genommen werden? Denn irgend eine mufs man offenbar haben. Die französischen Mathematiker, welche ich vergleichen konnte, sagen alle: man variire $\int d s . v$, aber nicht, nach was. Das Nächstliegende jedoch ist, wie überall sonst in der Mechanik, wo nichts Besonderes bemerkt ist, so auch hier, die Zeit $t$ als unabhängige Veränderliche anzunehmen. Geschieht aber dies, so ergiebt sich als der erste Uebelstand, dafs dann die Grenzen des Integrals von einer Curve zur andern sich gleichfalls ändern; denn die Endpuncte der Bahnen sind zwar gegeben, aber keinesweges die Zeit, in welcher sie durchlaufen werden. In diesem Falle - wäre also

$$
\delta \int d s . v \quad \text { nicht }=\int \delta(d s . v)
$$

wie es vorausgesetzt wird. Deswegen aber die Nachbarcurven, die beim Maximum oder Minimum in Betracht kommen können, auf diejenigen zu beschränken, die in gleicher Zeit durchlaufen werden, ist, wie sogleich gezeigt werden soll, unnöthig. Dann fragt es sich aber weiter, ob man den Zweck auch erreiche, wenn nach $t$ variirt wird. Es soll unter allen Curven diejenige gesucht werden, für welche $\int d s . v$ ein Minimum wird. Die Form dieser Curven aber ist ganz unabhängig von der Zeit, in welcher sie durchlaufen werden; und aufserdem würde man, wenn man nach $t$ variirte, auch noch verschiedene Arten der Bewegung in einer und derselben Curve erhalten; um die es sich aber hier nicht handelt. Um also diejenige Curve zu finden, für welche $\int d s . v$ ein Minimum wird, mufs man nicht nach $t$ variiren, sondern nach einem Ausdruck, der aus den Coordinaten der Bahn geometrisch zusammengesetzt ist, etwa einem Radius - vector, oder dergl.; wobei dann auch die Grenzen constant sind. Dies hat im vorliegenden Falle um so weniger Schwierigkeit, als der Voraussetzung gemäfs $v$ eine blofse Function von $x, y, z$, ohne $t$ explicit zu enthalten, und $s$ ohnehin eine geometrische Gröfse ist. Bezeichnet man also die aus $x$, $y, z$ geometrisch zusammengesetzte Function, nach welcher variirt werden soll, mit $u$, so läfst sich der Beweis folgendermafsen führen. Es ist

$$
\begin{aligned}
& \text { 1. } \int d s \cdot v=\int d u \cdot v \cdot \frac{d s}{d u}, \\
& \text { 2. } \delta \int d s \cdot v=\int d u \cdot\left(\frac{d s}{d u} \delta v+v \cdot \delta \frac{d s}{d u}\right),
\end{aligned}
$$


15. Zech, über das Princip der Kleinsten Wirkung.

$$
\left(\frac{d s}{d u}\right)^{2}=\left(\frac{d x}{d u}\right)^{2}+\left(\frac{d y}{d u}\right)^{2}+\left(\frac{d z}{d u}\right)^{2},
$$

3. $\frac{d s}{d u} \cdot \delta \frac{d s}{d u}=\frac{d x}{d u} \cdot \frac{d \delta x}{d u}+\frac{d y}{d u} \cdot \frac{d \delta y}{d u}+\frac{d z}{d u} \cdot \frac{d \delta z}{d u}$,

folglich, wegen

$$
\boldsymbol{v}=\frac{d s}{d t}=\frac{d s}{d u} \cdot \frac{d u}{d t},
$$

4. $v \cdot \delta \frac{d s}{d u}=\frac{d x}{d t} \cdot \frac{d \delta x}{d u}+\frac{d y}{d t} \cdot \frac{d \delta y}{d u}+\frac{d z}{d t} \cdot \frac{d \delta z}{d u}$.

Aus der Gleichung

$$
v^{2}=C+2 \int(X d x+Y d y+Z d z)
$$

folgt, wenn der Voraussetzung gemäls $X d x+Y d y+Z d z$ ein vollständiges Differential ist und nach $u$ variirt wird,

mithin wegen

$$
\text { 5. } v \cdot \delta v=\boldsymbol{X} \delta x+\boldsymbol{Y} \delta y+\boldsymbol{Z} \delta \boldsymbol{z}
$$

$$
\begin{aligned}
\frac{d s}{d u} & =\frac{d s}{d t} \cdot \frac{d t}{d u}=v \cdot \frac{d t}{d u}, \\
\text { 6. } \quad \frac{d s}{d u} \cdot \delta v & =\frac{d t}{d u}(X \delta x+Y \delta y+Z \delta z) .
\end{aligned}
$$

Substituirt man den Werth von $v . \delta \frac{d s}{d u}$ aus (4.) und den von $\frac{d s}{d \imath} \delta v$ aus (6.) in die (2.), so ergiebt sich

$\delta \int d s . v=\int_{u^{\prime}}^{u^{\prime \prime}} d u\left\{\frac{d t}{d u}(X \delta x+Y \delta y+Z \delta z)+\frac{d x}{d t} \cdot \frac{d \delta x}{d u}+\frac{d y}{d t} \cdot \frac{d \delta y}{d u}+\frac{d z}{d t} \cdot \frac{d \delta z}{d u}\right\}$, wenn $u^{\prime}$ und $u^{\prime \prime}$ die Werthe von $u$ an Anfang und am Ende der Bahn bezeichnen. Durch theilweises Integriren und wegen

erhält man hieraus

$$
\frac{d}{d u} \cdot \frac{d x}{d t}=\frac{d t}{d u} \cdot \frac{d^{2} x}{d t^{2}}
$$

$$
\begin{aligned}
\delta \int d s . v= & \left\{\frac{d x}{d t} \delta x+\frac{d y}{d t} \delta y+\frac{d z}{d t} \delta z\right\}_{u^{\prime \prime} \div u^{\prime}} \\
& +\int_{u^{\prime}}^{u^{\prime \prime}} d u \frac{d t}{d u}\left\{\left(X-\frac{d^{2} x}{d t^{2}}\right) \delta x+\left(Y-\frac{d^{2} y}{d t^{2}}\right) \delta y+\left(Z-\frac{d^{2} z}{d t^{2}}\right) \delta z\right\} .
\end{aligned}
$$

Nun ist aber an beiden Grenzen $\delta x=0, \delta y=0, \delta z=0$ und, nach bekanuten Gleichungen der Dynamik,

$$
X=\frac{d^{2} x}{d t^{2}}, \quad Y=\frac{d^{2} y}{d t^{2}}, \quad Z=\frac{d^{2} z}{d t^{2}} ;
$$

also ist

$$
\text { 7. } \delta \int d s \cdot v=0 \text {. }
$$


Daraus folgt nun aber noch keineswegs, wie gewöhnlich geschlossen wird, dafs $\int d s . v$ ein Maximum oder Minimum sei, sondern, um entscheiden zu können, ob überhaupt ein Maximum oder Minimum stattfinde, und im bejahenden Fall, welches von beiden, ist $\delta^{2} \int d s . v$ zu untersuchen. Aus der Gleichung (2.) folgt aber

$$
\text { 8. } \quad \delta^{2} \int d s \cdot v=\int d u\left(\frac{d s}{d u} \cdot \delta^{2} v+2 \delta v \cdot \delta \frac{d s}{d u}+v \cdot \delta^{2} \frac{d s}{d u}\right) \text {. }
$$

Variirt man die (3.) und (5.) noch einmal, so erhält man

$$
\begin{aligned}
& \text { 9. } \quad \frac{d s}{d u} \delta^{2} \frac{d s}{d u}+\left(\delta \frac{d s}{d u}\right)^{2} \\
& =\frac{d x}{d u} \cdot \frac{d \delta^{2} x}{d u}+\left(\frac{d \delta x}{d u}\right)^{2}+\frac{d y}{d u} \cdot \frac{d \delta^{2} y}{d u}+\left(\frac{d \delta y}{d u}\right)^{2}+\frac{d z}{d u} \cdot \frac{d \delta^{2} z}{d u}+\left(\frac{d \delta z}{d u}\right)^{2}, \\
& =X \delta^{2} x+\frac{d X}{d x}(\delta x)^{2}+\frac{d X}{d y} \delta x \cdot \delta y+\frac{d X}{d z} \delta x \cdot \delta z \\
& +Y \delta^{2} y+\frac{d Y}{d x} \delta x \cdot \delta y+\frac{d Y}{d y}(\delta y)^{2}+\frac{d Y}{d z} \delta y \cdot \delta z \\
& +Z \delta^{2} z+\frac{d Z}{d x} \delta x \cdot \delta z+\frac{d Z}{d y} \delta y \cdot \delta z+\frac{d Z}{d z}(\delta z)^{2} \\
& =X \delta^{2} x+Y \delta^{2} y+Z \delta^{2} z+\frac{d X}{d x}(\delta x)^{2}+2 \frac{d X}{d y} \delta x \cdot \delta y+2 \frac{d X}{d z} \delta x \cdot \delta z \\
& +\frac{d Y}{d y}(\delta y)^{2}+2 \frac{d Y}{d z} \delta y \cdot \delta z+\frac{d Z}{d z}(\delta z)^{2} .
\end{aligned}
$$

Jenn denkt man sich $\int(X d x+Y d y+Z d z)$ wirklich integrirt, so dars

$$
\int(\boldsymbol{X} d x+Y d y+Z d z)=F(x, y, z)
$$

ist, so hat man

$$
X=\frac{d F}{d x} ; \quad Y=\frac{d F}{d y} ; \quad Z=\frac{d F}{d z},
$$

also

$$
\frac{d X}{d y}=\frac{d Y}{d x} ; \quad \frac{d X}{d z}=\frac{d Z}{d x} ; \quad \frac{d Y}{d z}=\frac{d Z}{d y} .
$$

Die (9.) mit $\frac{d u}{d t}$ multiplicirt giebt

$$
\begin{gathered}
\text { 11. v. } \delta^{2} \frac{d s}{d u}+\frac{d u}{d t}\left(\delta \frac{d s}{d u}\right)^{2} \\
=\frac{d u}{d t}\left\{\frac{d x}{d u} \cdot \frac{d \delta^{2} x}{d u}+\left(\frac{d \delta x}{d u}\right)^{2}+\frac{d y}{d u} \cdot \frac{d \delta^{2} y}{d u}+\left(\frac{d \delta y}{d u}\right)^{2}+\frac{d z}{d u} \cdot \frac{d \delta^{2} z}{d u}+\left(\frac{d \delta z}{d u}\right)^{2}\right\} .
\end{gathered}
$$

Die (10.), mit $\frac{d t}{d \iota}$ multiplicirt, giebt 


$$
\begin{aligned}
& \text { 12. } \frac{d s}{d u} \cdot \delta^{2} v+\frac{d t}{d u}(\delta v)^{2} \\
& =\frac{d t}{d u}\left\{\boldsymbol{X} . \delta^{2} x+\boldsymbol{Y} \cdot \delta^{2} y+\boldsymbol{Z} . \delta^{2} z+\frac{d \boldsymbol{X}}{d x}(\delta x)^{2}+2 \frac{d \boldsymbol{Y}}{d y} \delta x \cdot \delta y+2 \frac{d \boldsymbol{X}}{d z} \delta x \cdot \delta z\right. \\
& \left.+\frac{d Y}{d y}(\delta y)^{2}+2 \frac{d Y}{d z} \delta y \cdot \delta z+\frac{d Z}{d z}(\delta z)^{2}\right\} .
\end{aligned}
$$

Wird nun in (11.) $\frac{d u}{d t}\left(\delta \frac{d s}{d u}\right)^{2}$ und in (12.) $\frac{d t}{d u}(\delta v)^{2}$ auf die rechte Seite gebracht, werden dann beide Gleichungen addirt und wird auf beiden Seiten der so entstehenden Gleichung noch $2 \delta v . \delta \frac{d s}{d u}$ hinzugefügt, so bekommt man

$$
\begin{aligned}
\text { 13. } \frac{d s}{d u} \cdot \delta^{2} v+2 \delta v \cdot \delta \frac{d s}{d u}+v \cdot \delta^{2} \frac{d s}{d u} \\
=-\left\{\frac{d t}{d u}(\delta v)^{2}-2 \delta v \cdot \delta \frac{d s}{d u}+\frac{d u}{d t}\left(\delta \frac{d s}{d u}\right)^{2}\right\} \\
+\frac{d t}{d u}\left(X \cdot \delta^{2} x+Y \cdot \delta^{2} y+Z \cdot \delta^{2} z\right)+\frac{d x}{d t} \cdot \frac{d \delta^{2} x}{d u}+\frac{d y}{d t} \cdot \frac{d \delta^{2} y}{d u}+\frac{d z}{d t} \cdot \frac{d \delta^{2} z}{d u} \\
+\frac{d t}{d u}\left\{\frac{d X}{d x}(\delta x)_{2}+2 \frac{d X}{d y} \delta x \cdot \delta y+2 \frac{d X}{d z} \delta x \cdot \delta z+\frac{d Y}{d y}(\delta y)^{2}+2 \frac{d Y}{d z} \delta y \cdot \delta z\right. \\
\left.+\frac{d Z}{d z}(\delta z)^{2}+\left(\frac{d \delta x}{d t}\right)^{2}+\left(\frac{d \delta y}{d t}\right)^{2}+\left(\frac{d \delta z}{d t}\right)^{2}\right\} .
\end{aligned}
$$

Nun ist aber

$$
\begin{gathered}
\frac{d t}{d u}(\delta v)^{2}-2 \delta v \cdot \delta \frac{d s}{d u}+\frac{d u}{d t}\left(\delta \frac{d s}{d u}\right)^{2}=\frac{d t}{d u}\left(\delta v-\frac{d u}{d t} \cdot \delta \frac{d s}{d u}\right)^{2} \\
=\frac{d t}{d u}\left(\delta v-\delta \frac{d s}{d t}\right)^{2}=0,
\end{gathered}
$$

$\int_{u^{\prime}}^{t^{\prime \prime \prime}} d u \cdot\left\{\frac{d t}{d u}\left(\boldsymbol{X} \cdot \delta^{2} x+\boldsymbol{Y} \cdot \delta^{2} y+Z \cdot \delta^{2} z\right)+\frac{d x}{d t} \cdot \frac{d \delta^{2} x}{d u}+\frac{d y}{d t} \cdot \frac{d \delta^{2} y}{d u}+\frac{d z}{d t} \cdot \frac{d \delta^{2} z}{d u}\right\}$ $=\left\{\frac{d x}{d t} \delta^{2} x+\frac{d y}{d t} \delta^{2} y+\frac{d z}{d t} \delta^{2} z\right\}_{u^{\prime \prime} \div u^{\prime}}$

$$
+\int d u \cdot \frac{d t}{d u}\left\{\left(X-\frac{d^{2} x}{d t^{2}}\right) \delta^{2} x+\left(Y-\frac{d^{2} y}{d t^{2}}\right) \delta^{2} y+\left(Z-\frac{d^{2} z}{d t^{2}}\right) \delta^{2} z\right\}
$$

$=0$.

Aus den beiden letztern Gleichungen, in Verbindung mit (13.) und (8.), folgt 14. $\delta^{2} \int d s . v=\int d t\left\{\left(\frac{d X}{d x}(\delta x)^{2}+2 \frac{d X}{d y} \delta x . \delta y+2 \frac{d X}{d z} \delta x \cdot \delta z+\frac{d Y}{d y}(\delta y)^{2}\right.\right.$

$$
\left.+2 \frac{d Y}{d z} \delta y \cdot \delta z+\frac{d Z}{d z}(\delta z)^{2}+\left(\frac{d \delta x}{d t}\right)^{2}+\left(\frac{d \delta y}{d t}\right)^{2}+\left(\frac{d \delta z}{d t}\right)^{2}\right\} .
$$

In jedem besonderen Falle mufs also noch untersucht werden, ob dieser Ausdruck nicht der Null gleich und ob er stels positiv oder stets negativ sei. Für die freie Bewegung in der Parabel hat mau 
mithin

$$
\boldsymbol{X}=0, \quad \boldsymbol{Y}=-\boldsymbol{g}, \quad \boldsymbol{Z}=0,
$$

$\frac{d X}{d x}=0, \quad \frac{d X}{d y}=0, \quad \frac{d X}{d z}=0, \quad \frac{d Y}{d y}=0, \quad \frac{d Y}{d z}=0, \quad \frac{d Z}{d z}=0$, folglich

$$
\delta^{2} \int d s . v=\int d t\left\{\left(\frac{d \delta x}{d t}\right)^{2}+\left(\frac{d \delta y}{d t}\right)^{2}+\left(\frac{d \delta z}{d t}\right)^{2}\right\} \text { stets }>0,
$$

also wirklich $\int d s . v$ ein Minimum.

Wie stimmt nun aber dies mit dem oben berechneten Beispiele? Erinnern wir uns hier, dafs aus den Gleichungen

$$
\delta \boldsymbol{V}=0 \text { und } \delta^{2} \boldsymbol{V}>0
$$

nur dann geschlossen werden kann, $V$ sei ein Minimum, wenn die Grölse, nach deren steigenden Potenzen die variirte Function $\boldsymbol{V}$ fortlauft, so klein ist, dafs jedes Glied der Reihe maarsgebend ist für das Zeichen der. Summe der Glieder von ihm an abwärts. Das Princip der kleinsten Wirkung gilt also nur in Beziehung auf die nüchst anliegenden Curven, und obiges Beispiel gehört gar nicht in die' Kategorie desselben.

Zu einer weitern Beschränkung des Princips, als den beiden eben angeführten, nämlich dafs $\delta^{2} \int \boldsymbol{d} s . v$ zuvor untersucht werden mufs, und dafs nur nächst - anliegende Curven in Betracht kommen können, etwa die Grölse oder Richtung der Anfangsgeschwindigkeit in den Nachbarcurven betreffend, ist in dem Beweise selbst kein Grund vorhanden.

Die Anwendung endlich, die schon von dem Princip der kleinsten Wirkung gemacht worden ist, um für gegebene Kräfte die Curve der freien Bewegung zu finden, ist durch das bisher Bemerkte nicht ausgeschlossen. Für den Fall der freien Bewegung ist nach wie vor stets $\delta \int d s . v=0$. So lange aber nicht bewiesen ist, dafs nur in Falle der freien Bewegung $\delta \int \boldsymbol{d} s . v=0$ ist, bleiben die auf jene Art gefundenen Resultate immer nur problematisch.

Tübingen, den 31. Juli 1841. 

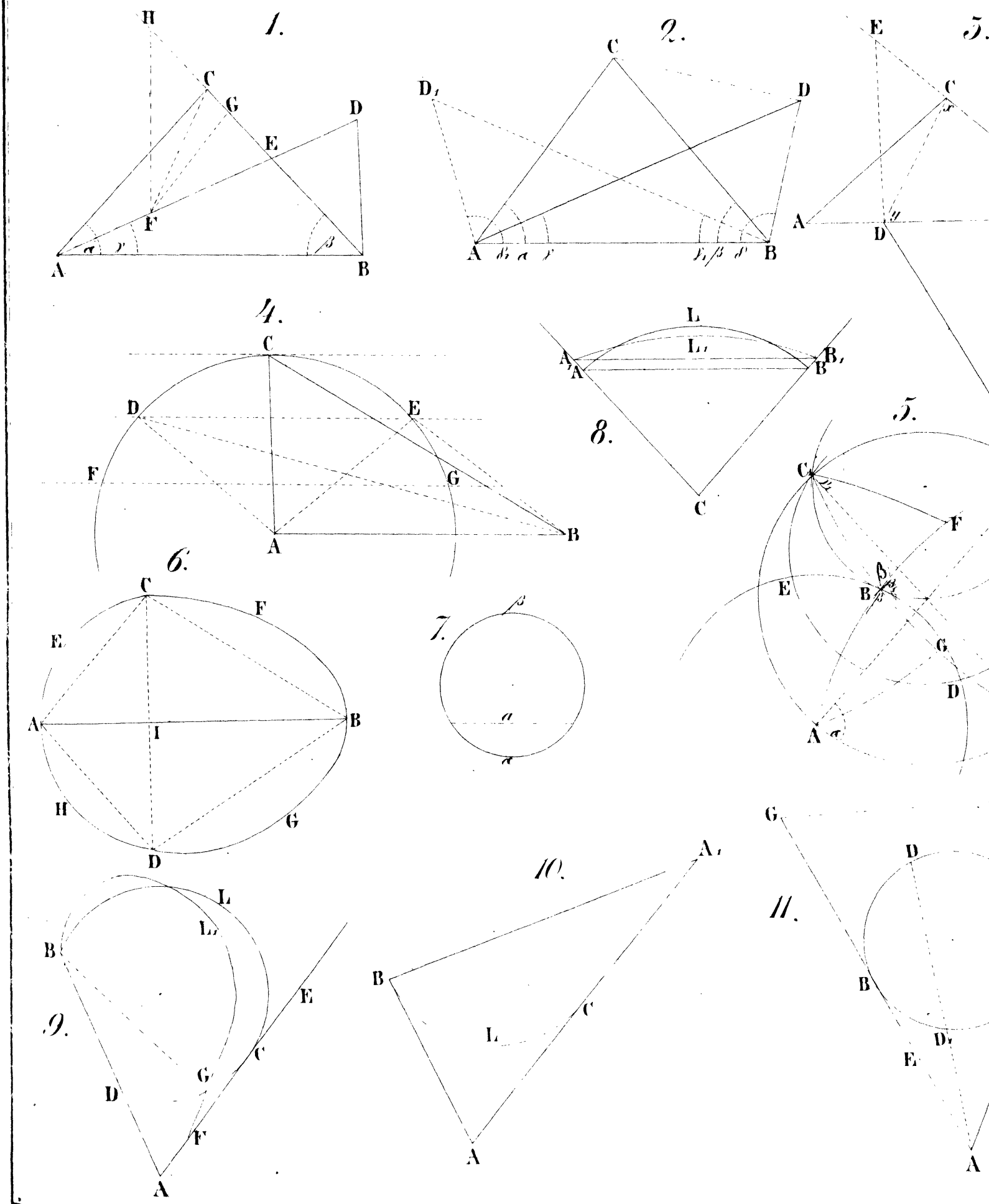

B
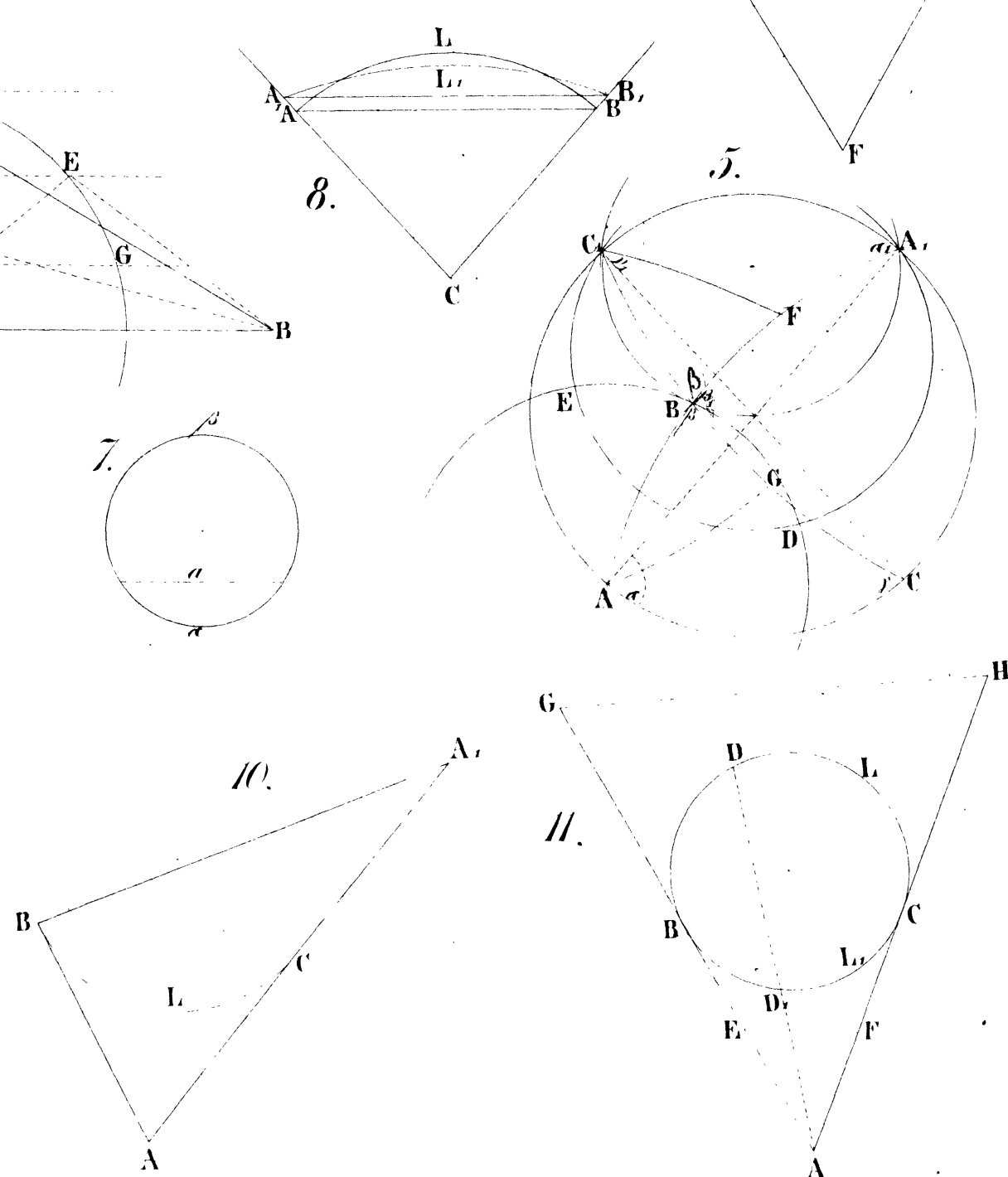

8.

${ }_{B} \mathrm{~A}$

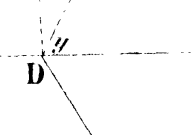

. 


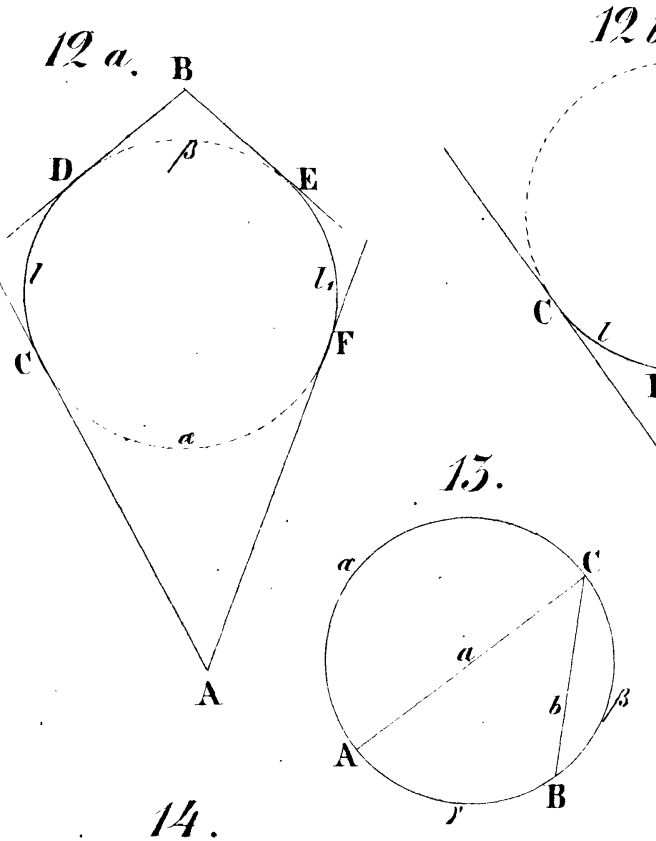

?b.
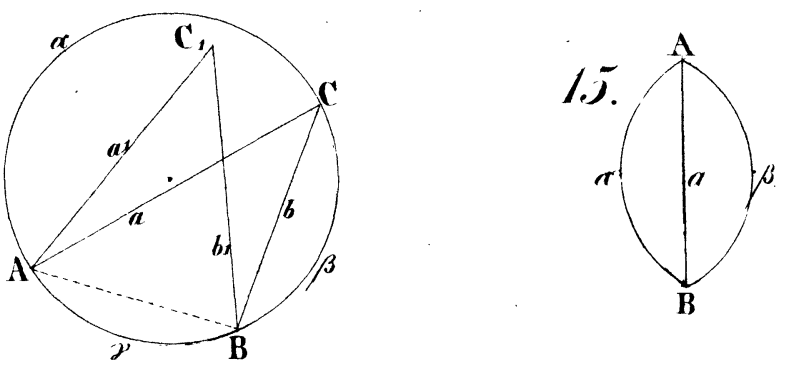
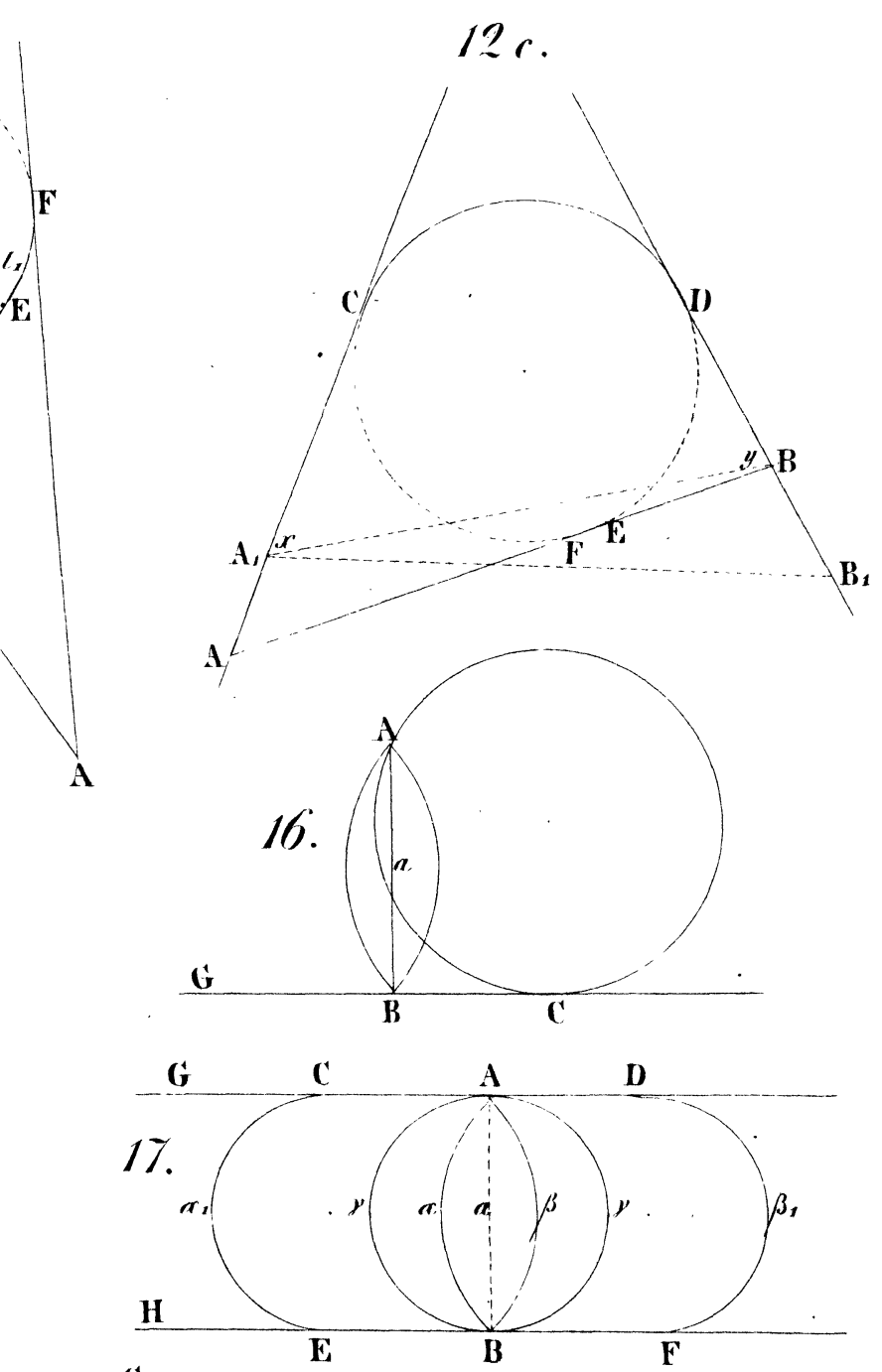

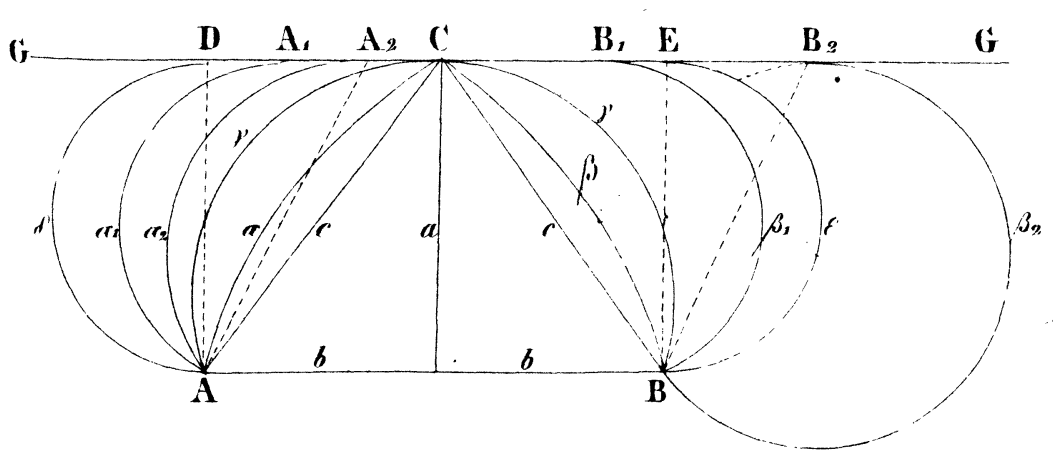


Brought to you by | University of Iowa Libraries Authenticated

Download Date | 6/21/15 10:44 AM 
Girelle, Sournal d Wrath bir.XXVVHifl 2.

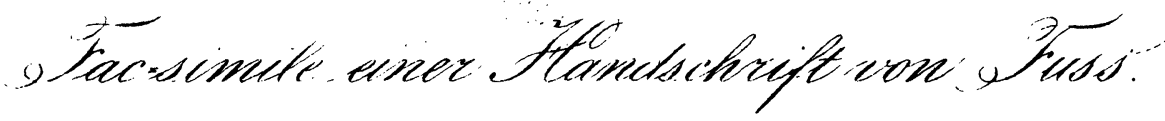

$$
\begin{aligned}
& \text { Sunimatió Seriei } \\
& \left.P=\frac{z^{3}}{1.3}-\frac{z^{7}}{1.5 .5 .7}+\frac{z^{11}}{1 \cdots 11}-\frac{z^{1.50}}{1 \cdots 1.5}+2 x\right)
\end{aligned}
$$

Differentiando erit

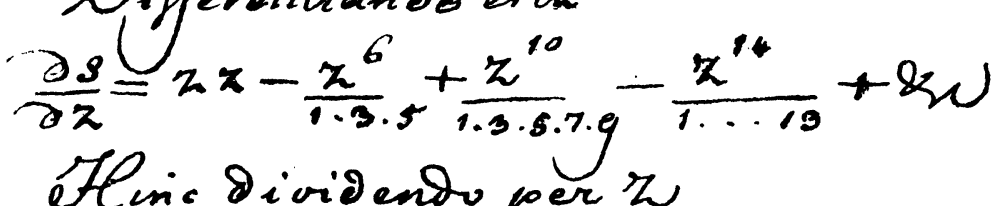

Éfiric dividendu per $z$

$$
\frac{\partial s}{2 \partial z}=z-\frac{z^{5}}{1 \cdot 3 \cdot 5^{5}}+\frac{z^{g}}{1 \cdot 3 \cdot 5 \cdot 7 \cdot g}-\frac{z^{13}}{1 \cdot 1 \cdot 19}+2 z 0
$$

remeogs oifferentixato.

$$
\partial \cdot \frac{\partial \rho}{\partial \partial z^{2} .1} 1-\frac{z^{4}}{1.3}+\frac{z^{8}}{1 \cdot 3 \cdot 5 \cdot 7}-\frac{z^{12 .}}{1 \cdot 3 \cdot 5 \cdot 7 \cdot y^{11}}+820
$$

unde manifestuon est fore

$$
\frac{\partial \rho}{z \partial z^{2}}=1-s z
$$

Atanc arequationem facile irito grare licoret, firinoo unitaco abefoct. Ca enim onifor forat $\partial \cdot \frac{\partial s}{\partial \partial z^{\prime \prime}}=-s z$, qual in $\frac{\partial s}{\partial \partial z^{2}} \partial u$ ta \& in êgrata pracbect

$$
\frac{\partial s^{2}}{2 z \partial z^{4}}=e x-\frac{\rho g}{\partial z^{2}}
$$

five hownoyencitaly gratia

$\frac{\partial s}{z 2 \partial z^{4}}=\frac{a a-s s}{\partial z^{2}}$

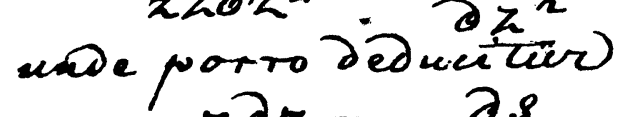

$$
z \partial x=\frac{\partial s}{\sqrt{a a-s s}}
$$

hineque integrañ.

$$
\frac{1}{a} z z=\operatorname{lithin}_{a}
$$

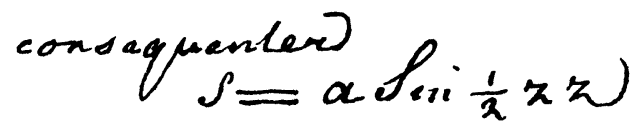

Vunc consideremus aeguationein niostrain ว. $\frac{\partial S}{\partial \partial z^{a}}=1 \ldots s$ 政

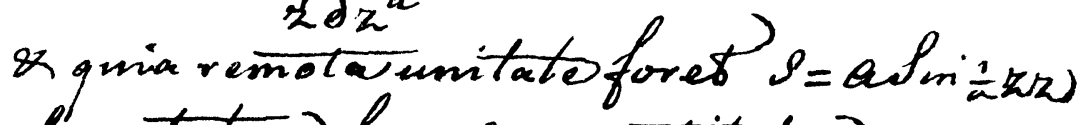
ob unitatem low a gpantitatem varia. bilam introd ucemuls, pon endo

$s=t \sin \frac{1}{a}$ ro

aritgue diffarentiond.

$\partial s=\partial t \rho_{\min \frac{1}{n} z} z+t_{z} \partial z \cos \frac{1}{2} \pi z$

consequenter

$\frac{\partial g}{z \partial z}=\frac{\partial t}{z \partial z} \rho_{m} \frac{1}{\lambda} z z+t \cos i z z$

renicoquevifferentianos

$\partial \cdot \frac{\partial S}{\partial \partial z^{2}}=\frac{\partial \partial t}{\partial \partial z^{2}} \sin \frac{1}{\pi} \pi z+\frac{\partial \partial t}{\partial z} \cos \frac{1}{\pi} \pi \omega$

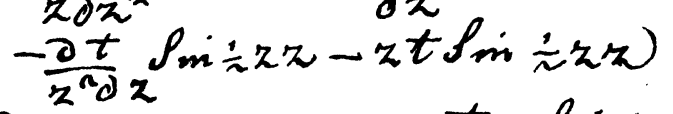

Esvero $\partial \cdot \frac{\partial s}{\partial \partial z^{2}}=1-s z=1-t z \sin \frac{1}{n} z z$ conseq enter erit

$\frac{\partial \partial t}{\pi \partial} \sin \frac{1}{n} z z+2 \partial t \cos \frac{1}{\pi} \pi z-\frac{\partial t}{z z} \sin \frac{1}{\pi} z z=\partial z$.

Sonatur $\partial t=u \partial z$, fietgre

$\frac{z \partial u}{z z} \sin \div z z+z u \partial z \cos \frac{1}{\pi} z z-\frac{\omega \partial z}{z} \sin \frac{1}{\pi} z z=\partial z$.

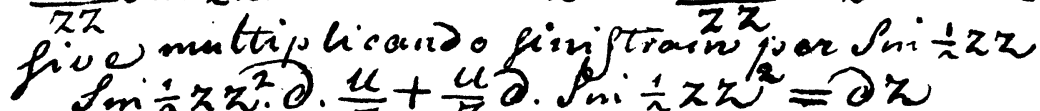

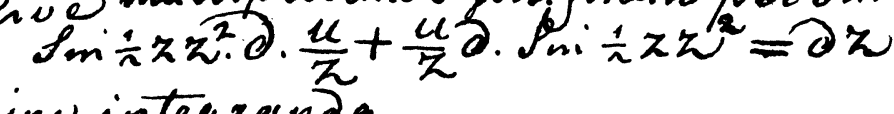

lime integreento

$$
\begin{aligned}
& \frac{u}{z} \sin \frac{1}{\pi} \pi x^{a}=\pi \\
& \pi=\frac{\partial t}{\partial z}=\frac{\pi z}{f_{\text {in }} \cdot \frac{\pi}{\pi} \pi z^{a}} \\
& \partial t=\frac{z z \partial x}{\rho_{\min } \frac{\dot{\lambda} \pi z^{2}}{\pi}}
\end{aligned}
$$

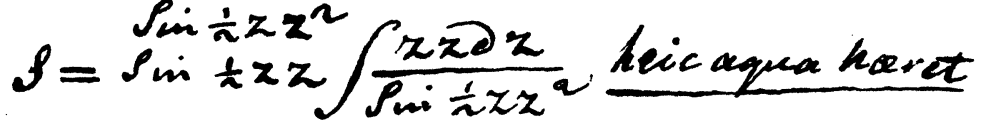


Brought to you by | University of lowa Libraries Authenticated 